\title{
Nucleolar spindle associated protein 1 (NUSAP1) facilitates proliferation of hepatocellular carcinoma cells
}

\author{
Sinan Hou ${ }^{1,2}$, Lei Hua ${ }^{3}$, Weidong Wang $^{1}$, Mengjie $\mathrm{Li}^{3}$, Linfeng Xu${ }^{1}$ \\ ${ }^{1}$ Department of Interventional Radiology, ${ }^{2}$ Guangdong Provincial Key Laboratory of Malignant Tumor Epigenetics and Gene Regulation, Sun \\ Yat-sen Memorial Hospital, Sun Yat-sen University, Guangzhou 510120, China; ${ }^{3}$ Department of Pain Management, Wuhan Fourth Hospital, Puai \\ Hospital, Tongji Medical College, Huazhong University of Science and Technology, Wuhan 430033, China \\ Contributions: (I) Conception and design: All authors; (II) Administrative support: L Xu; (III) Provision of study materials or patients: W Wang; (IV) \\ Collection and assembly of data: S Hou, L Hua, M Li; (V) Data analysis and interpretation: S Hou, L Hua, W Wang; (VI) Manuscript writing: All \\ authors; (VII) Final approval of manuscript: All authors. \\ Correspondence to: Linfeng Xu. Department of Interventional Radiology, Sun Yat-sen Memorial Hospital, Sun Yat-sen University, No. 107 Yanjiang \\ Road West, Guangzhou 510120, China. Email: xulf@mail.sysu.edu.cn.
}

Background: The most fundamental characteristic of cancer cells is abnormal proliferation, which is partly due to deregulation of cell cycle. Nucleolar spindle associated protein 1 (NUSAP1) is originally identified as microtubule and chromosome binding protein and takes an important role in chromosome segregation fidelity. Recent studies have been documented that NUSAP1 is associated with the progression of several cancers. However, its expressions and functions are unclear in hepatocellular carcinoma (HCC).

Methods: qPCR and western blotting were used to examine NUSAP1 levels in fresh paired HCC tissues and corresponding adjacent normal liver tissues (ANT). MTT and colony formation assay were performed to evaluate whether NUSAP1 can influence the proliferation of HCC cells. Anchorage-independent growth assay was implemented to detect the effect of NUSAP1 on in vitro tumorigenicity capacity. Flow cytometry, qPCR and western blotting assay were used to clarify the detailed mechanisms that NUSAP1 accelerates the capability for cell proliferation and in vitro tumorigenicity.

Results: Firstly, we analyzed the availably public dataset The Cancer Genome Atlas (TCGA), and discovered that NUSAP1 is dramatically upregulated in HCC tissues and closely correlated with poor prognosis of patients suffering HCC, which was confirmed in fresh HCC tissues and ANT. Subsequently, cell function assay illustrated that the upregulation of NUSAP1 facilitates proliferation and in vitro tumorigenicity capacity of HCC cells. Besides, molecular experiments suggested that NUSAP1 promotes cell cycle transition of HCC cells.

Conclusions: In summary, the above results inferred that NUSAP1 takes an indispensable role in cell cycle progression. And it might be served as a novel prognostic factor in HCC.

Keywords: Cell cycle transition; hepatocellular carcinoma (HCC); proliferation; nucleolar spindle associated protein 1 (NUSAP1)

Submitted May 19, 2019. Accepted for publication Aug 28, 2019.

doi: $10.21037 /$ tcr.2019.09.28

View this article at: http://dx.doi.org/10.21037/tcr.2019.09.28

\section{Introduction}

Liver cancer is evaluated to be the sixth most commonly diagnosed cancer and the fourth leading reason of cancer related death worldwide in 2018 , with approximate 841,000 new cases and 782,000 deaths yearly. Among liver cancer, $78-85 \%$ of cases are hepatocellular carcinoma (HCC) (1). Therefore, it is essential to investigate the detailed mechanisms of tumorigenicity in HCC.

The most fundamental characteristic of cancer cells 
is abnormal proliferation (2), which is partly caused by misregulation of cell cycle. The cell cycle of eukaryotic cells includes four phase, namely, G1, S, G2 and M. The phase transition is critically driven by cyclin-dependent kinases (CDKs). As the central regulatory determinant in cell cycle, CDKs are suffered to many levels of regulation, and their activities change dependent on both intracellular and extracellular environment $(3,4)$. The first level of regulation is implemented by cyclins, and another level is by CDKs inhibitors (CKIs) to inhibit phosphorylation of CDKs. Of which, cyclin abundance oscillates during the cell cycle as a result of programmed synthesis and degradation. The G1/ $S$ phase transition is driven by CDK2, CDK4, CDK6 and corresponding cyclins. CDK4 and CDK6 are regulated by D-type cyclins: cyclin D1, cyclin D2 and cyclin D3. Cyclin E accumulates very closely to G1/S transition and specifically activates CDK2. Activated CDKs can target and phosphorylate retinoblastoma (p-Rb). p-Rb is identified to be the first suppressor gene. It can repress transcription through binding transactivation domain of E2F family (5). Phosphorylated $\mathrm{p}-\mathrm{Rb}$ alleviates its inhibition on E2F family and allows the transcription of genes essential for $\mathrm{S}$ phase entry and DNA synthesis (6). CDK1 or CDK2 together with cyclin $\mathrm{A}$ are indispensable for the continuation of $\mathrm{S}$ phase and entry into $M$ phase. CDK1 together with cyclin $B$ also promote entry into $M$ phase, and cyclin $\mathrm{A}$ is degraded before cyclin B by the proteasome (7). The intracellular and extracellular signals that affect phase transition of cell cycle play crucial roles in cell cycle progression. Therefore, we aim to investigate the factors that affect the cell cycle transition.

Nucleolar spindle associated protein 1 (NUSAP1) is originally identified as microtubule and chromosome binding protein. It takes an important role in chromosome segregation fidelity (8-10). Recently, it has been documented that NUSAP1 is abnormal expression, which is associated with poor prognosis in astrocytoma (10), prostate cancer (11), oral squamous cell carcinoma (12), prostate cancer (13), and breast cancer (14). However, its expressions and functions are unclear in HCC.

Herein, we found the expression of NUSAP 1 significantly elevates and is associated with poor prognosis in HCC. Further assay suggested that upregulation of NUSAP1 can promote cell cycle progression of HCC cells. The above results demonstrate that NUSAP1 might be a prognostic marker for HCC.

\section{Methods}

\section{Cell line and tissue specimens}

HCC cell HepG2 was purchased from American Type Culture Collection (ATCC) (Manassas, VA, USA) and cultured using Dulbecco's modified eagle medium (DMEM) (Invitrogen, Carlsbad, CA, USA) that contained 10\% fatal bovine serum (FBS) (HyClone, UT, USA). The primary HCC tissues and ANT were collected from Sun Yat-sen Memorial Hospital. For research purpose, we acquired the informed consent of patient involved in the research. This study was approved by institutional ethics committee of Sun Yat-sen Memorial Hospital, Sun Yat-sen University (No. W2017NJ07). The research has been carried out in accordance with the Declaration of Helsinki [2008] of the World Medical Association.

\section{Plasmids and retroviral infection}

For overexpression of NUSAP1, the full-length human NUSAP1 cDNA was amplified and cloned into the pMSCV vector. For downregulation of NUSAP1, 2 human siRNA sequences that target NUSAP1 were sub-cloned into pSuper-retro-puro plasmid. And the siRNA fragments are as follows: Sh\#1: GAGCACCAAGAAGCTGAGAAT; Sh\#2: GAACCACACAAAGGAAAGCTA. Infection was conducted using retrovirus. To get stable cell lines, we treated the cells using $0.5 \mu \mathrm{g} / \mathrm{mL}$ puromycin. 10 days later, we detected the levels of NUSAP1 in cells.

\section{Quantitative PCR (qPCR)}

We used the Trizol reagent (Invitrogen, Carlsbad, CA, USA) to collect total RNA. qPCR was performed using ABI 7500 Fast System (Applied Biosystems, Rockville, MD, USA). We evaluated the relative mount of each gene on the basis of the algorithm: $2^{-[(\mathrm{Ct} \text { of gene)-(Ct of } \beta \text {-Tubulin)] }}$. In the algorithm, the $\mathrm{Ct}$ is defined as threshold cycle and we used $\beta$-Tubulin as the internal control. The following are the primers of genes: NUSAP1: forward, 5'-CTGACCAAGACTCCAGCCAGAA-3', reverse, 5'-GAGTCTGCGTTGCCTCAGTTGT-3'; Cyclin D1: forward, 5'-TCTACACCGACAACTCCATCCG-3', reverse, 5'-TCTGGCATTTTGGAGAGGAAGTG-3'; Cyclin D3: forward, 5'-AGATCAAGCCGCACATGCGGAA-3', reverse, 5'-ACGCAAGACAGGTAGCGATCCA-3'; Cyclin E1: 
forward, 5'-TGTGTCCTGGATGTTGACTGCC-3', reverse, 5'-CTCTATGTCGCACCACTGATACC-3'; Cyclin A2: forward, 5'-CTCTACACAGTCACGGGACAAAG-3', reverse, 5'-CTGTGGTGCTTTGAGGTAGGTC-3'; Cyclin B1, forward, 5'-GACCTGTGTCAGGCTTTCTCTG-3', reverse, 5'-GGTATTTTGGTCTGACTGCTTGC-3'.

\section{Western blotting assay}

Western blotting assay was carried out on the basis of methods described (15). Whereafter, the membrane was probed by the primary antibodies: anti-NUSAP1, anti-p$\mathrm{Rb}$, anti- $\beta$-Tubulin (Abcam, Cambridge, MA, USA), and subsequently probed using HRP-conjugated secondary antibody (Abcam, Cambridge, MA, USA). And we used $\beta$-Tubulin as the loading control.

\section{3-(4,5-Dimethyl-2-thiazolyl)2,5-diphenyl-2H- tetrazolium bromide (MTT) assay}

$2 \times 10^{3}$ cells were seeded into 96 -well plates, subsequently maintained in $37^{\circ} \mathrm{C}$ incubator until indicated time. Whereafter, the cells were treated by $100 \mu \mathrm{L} 0.5 \mathrm{mg} / \mathrm{mL}$ sterile MTT reagent (sigma, St Louis, MO, USA) for 4 hours. And then, we added $150 \mu \mathrm{L}$ dimethyl sulphoxide (DMSO) (sigma, St Louis, MO, USA) into the plates. And the absorbance of cells was detected at $490 \mathrm{~nm}$.

\section{Colony formation assay}

Colony formation was used to evaluate the effect of NUSAP1 on proliferation of HCC cells and carried out on the basis of the methods described (16). $5 \times 10^{2}$ cells were implanted into 6-well plates and maintained in $37{ }^{\circ} \mathrm{C}$ incubator for 10 days. Firstly, we used 10\% formaldehyde to fix the colonies. And then, $1 \%$ crystal violet was applied to stain the colonies for 30 seconds.

\section{Anchorage-independent growth assay}

The completed medium that contains $1 \%$ agar (sigma, St Louis, MO, USA) was supplemented into the bottom of the cell plates. Then, the mixture that was composed of 500 cells, $0.3 \%$ agar and $2 \mathrm{~mL}$ complete medium were added into the upper of the plates. The cells were maintained in $37{ }^{\circ} \mathrm{C}$ incubator for 10 days and then the colonies with diameter larger than $0.1 \mathrm{~mm}$ were counted.

\section{Flow cytometry assay}

We collected the cells and fixed with $75 \%$ ethanol. Then, we applied $20 \mu \mathrm{g} / \mathrm{mL}$ RNase A and $50 \mu \mathrm{g} / \mathrm{mL}$ propidium iodide to treat the cells for 30 minutes away from light. The cells-treated were detected using FASCS alibur instrument (BD Biosciences, Franklin Lake, New Jersey, USA). Besides, CellQuest 3.3 software was used to evaluate the cells percentages in different phases.

\section{Statistical analysis}

Every experiment was repeated at least 3 times. SPSS 20.0 statistical software package was used to perform statistical analysis. The statistical significance between groups was evaluated by Student's $t$-test, and the data was demonstrated as mean \pm deviation. Survival curves were plotted using the Kaplan-Meier method, and significant analysis was using logrank test. $\mathrm{P}<0.05$ was considered as statistical significance.

\section{Results}

\section{NUSAP1 is significantly upregulated and associated with poor survival in $\mathrm{HCC}$}

Through analyzing the availably public dataset The Cancer Genome Atlas (TCGA) (https://cancergenome. nih.gov/), the results showed that NUSAP1 expression is significantly upregulated in $351 \mathrm{HCC}$ tissues compared with 50 normal liver tissues (Figure 1A). Subsequently, we further evaluated the NUSAP1 expression in 50 paired tissues. As demonstrated in Figure 1B, NUSAP1 is drastically upregulated in 47 HCC tissues compared with matched adjacent normal liver tissues (ANT). Besides, the correlation between NUSAP1 expression and clinical pathologic characteristics were performed. NUSAP1 expression is significantly upregulated in AJCC stage IIIIV compared with that in stage I-II (Figure 1C). Not only that, the patients with high-expression of NUSAP1 showed poorer 5-year overall survival and relapse-free survival than that with low NUSAP1 (Figure 1D).

We also check the NUSAP1 expression in 8 paired fresh HCC tissues and ANT using qPCR and western blotting assay. The results demonstrated that NUSAP1 expression is drastically elevated in HCC tissues than in ANT both on transcription and translation levels (Figure 2).

Altogether, NUSAP1 is significantly upregulated in HCC tissues and associated with poor prognosis. 


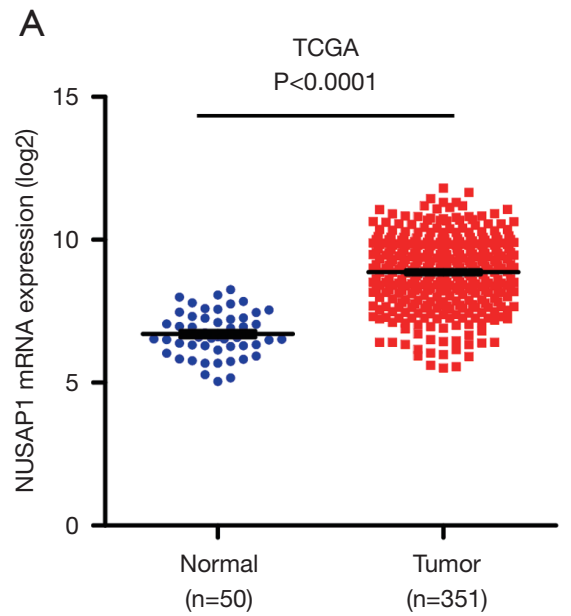

C TCGA AJCC pathologic_Stage

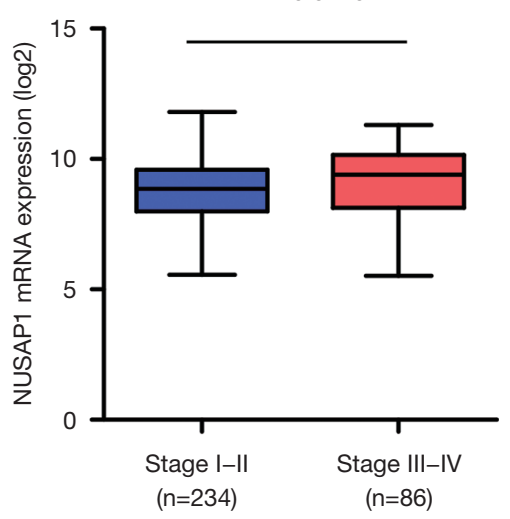

B

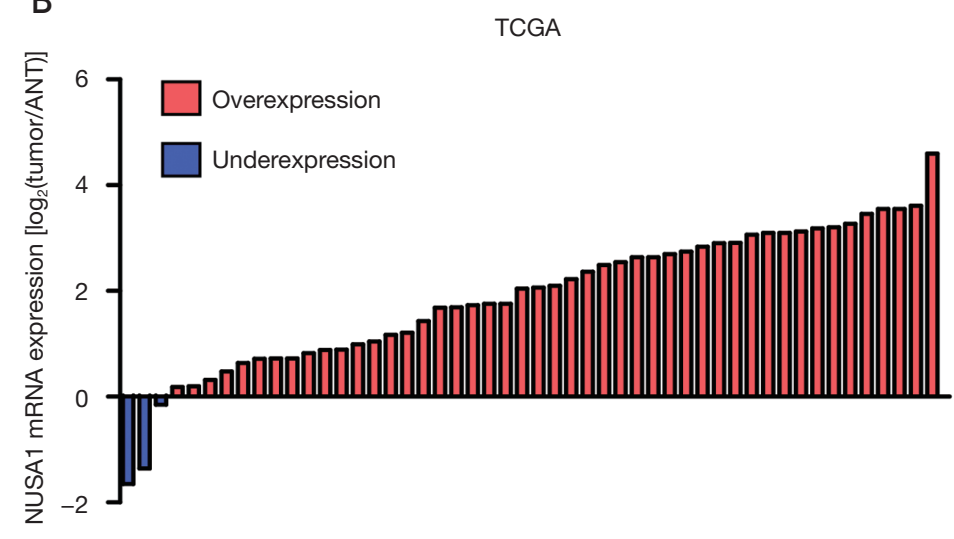

D

TGCA

Overall survival

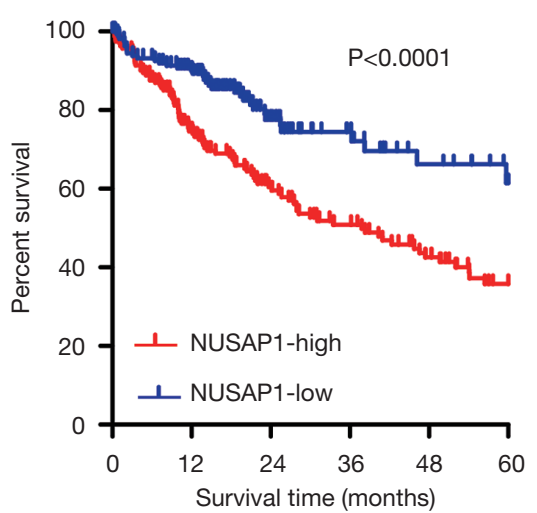

TGCA

Relapse-free survival

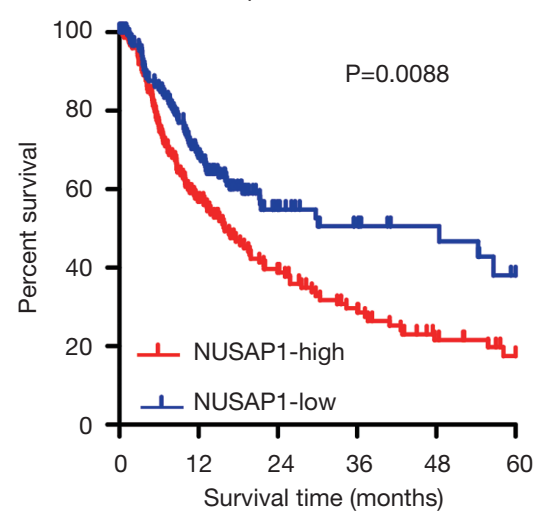

Figure 1 NUSAP1 expression is significantly upregulated in HCC by analyzing TCGA. (A) The NUSAP1 mRNA expression is drastically upregulated in HCC tissues compared with normal liver tissues. (B) The NUSAP1 mRNA expression in 50 paired tissues. (C) NUSAP1 mRNA expression is significantly upregulated in HCC stage III-IV compared with in stage I-II. (D) Kaplan-Meier 5-year overall survival (left panel) and relapse-free survival (right panel) curves for patients with high and low NUSAP1. NUSAP1, nucleolar spindle associated protein 1; HCC, hepatocellular carcinoma; TCGA, The Cancer Genome Atlas.

\section{Overexpression of NUSAP1 promotes the proliferation and in vitro tumorigenicity capacity of HCC cells}

To investigate the functions of NUSAP 1 in HCC progression, the gene set enrichment analysis (GSEA) was carried out using the data from TCGA. The analysis illustrated that NUSAP1 might be involved in cell cycle progression (Figure 3A). Next, the NUSAP1-overexpressed and -silenced stable cell lines were screened using HCC cell HepG2. The mRNA and protein expression of NUSAP1 was examined in the stable cell lines (Figure 3B). Next, MTT and colony formation assay demonstrated that overexpression of NUSAP1 significantly promoted HCC cells proliferation, and knockdown of NUSAP1 drastically inhibited cell proliferation (Figure 3C,D). Besides, anchorage-independent growth assay illustrated that in vitro tumorigenicity capacity of HCC cells promotes in NUSAP1-upregulating cells, while inhibits in NUSAP1downregulating cells (Figure 3E).

Altogether, overexpression of NUSAP 1 promoted proliferation and in vitro tumorigenicity capacity of HCC cells.

\section{NUSAP1 facilitates cell cycle transition in HCC cells}

To further explore the mechanisms by which NUSAP1 promotes cell proliferation and in vitro tumorigenicity capacity, GSEA was implemented. The analysis 

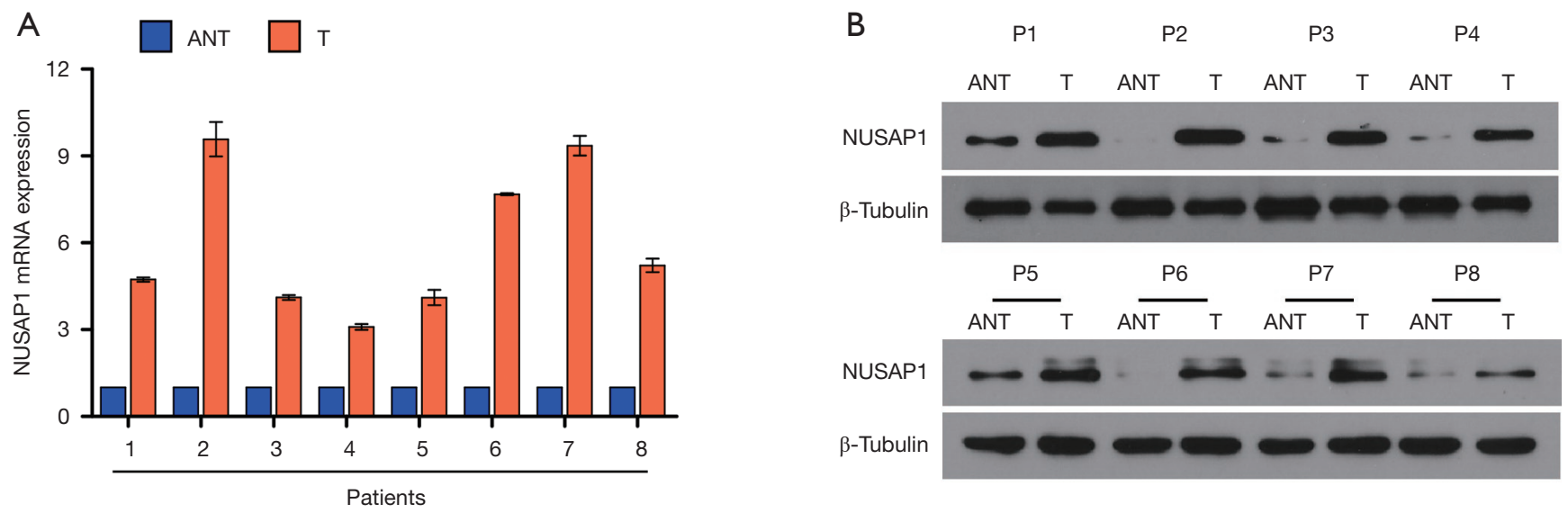

Figure 2 NUSAP1 expression is drastically upregulated in fresh HCC tissues compared with ANT by qPCR (A) and Western blotting assay (B). NUSAP1, nucleolar spindle associated protein 1; HCC, hepatocellular carcinoma; ANT, adjacent normal liver tissues.

demonstrated that NUSAP1 might be involved in G1/S and G2/M phase transition (Figure $4 A$ ). Next, flow cytometric assay was performed. As shown in Figure 4B, overexpression of NUSAP1 increases the percentage of cells in S-phase, but decreases cells in G0/G1 phase. While, downregulation of NUSAP1 showed the opposite results (Figure 4B). Not only that, we evaluated the mRNA expression of cyclins involved in G1/S and G2/M phase transition, cyclin D1, cyclin D3, cyclin E1, cyclin A2, cyclin B1 and the expression of E1F2. The heatmap illustrated that overexpression of NUSAP1 significantly increases their expression and downregulation of NUSAP1 inhibits their expression (Figure 4C). Besides, the phosphorylation level of $\mathrm{p}-\mathrm{Rb}$ is the critical determinant of G1/S phase transition. Therefore, we evaluated the phosphorylation level of $\mathrm{p}-\mathrm{Rb}$ by western blotting assay. As shown in Figure 4D, the phosphorylation level of $\mathrm{p}-\mathrm{Rb}$ enhances in NUSAP 1 -upregulated cells, while downregulates in NUSAP1-silenced cells, indicating that NUSAP1 can help to G1/S transition.

Altogether, overexpression facilitates cell cycle G1/S and G2/M phase transition of HCC cells.

\section{Discussion}

Herein, we discovered that the level of NUSAP 1 dramatically elevates in HCC tissues and is negatively related with prognosis of patients with HCC. Further analysis demonstrated that upregulation of NUSAP1 promotes the proliferation and in vitro tumorigenicity capacity of HCC cells and facilitates the G1/S and G2/M phase transition.

Aberrant proliferation of cells results in loss of the normal tissue architecture and functions, and the cancer cells obtain the capability for aberrant proliferation. Aberrant proliferation can be caused by multiple intracellular and extracellular signals. It has been reported that IncRNA FAL1 is up-regulated in HCC tissues and accelerates cell proliferation (17). Lin et al. showed that Exportin-T can promote cell proliferation through affecting cell cycle and ubiquitin-mediated proteolysis of HCC cells (18). Liu et al. clarified that PNMA1 significantly elevates in HCC tissues and is associated with poor prognosis (19). Shi and her colleagues suggested that TNNT1 is significant upregulated in breast cancer cells, and upregulation of TNNT1 can promote G1/S transition of breast cancer cells. Especially, TNNT1 can increase the levels of cyclin D1, cyclin E1, cyclin E2 and the phosphorylation of $\mathrm{Rb}$ (16). In particular, in consideration of CDKs ultimately driving the cell cycle transition, substantial preclinical test on CKIs are underway. Whereas, the results of clinical trials are disappointing (20). Our study might provide a novel idea on targeting cell cycle. NUSAP1 is upregulated to accelerate cell proliferation in HCC and essential for the integrity of the anaphase spindle and cell division. We speculated that targeting NUSAP1 can lead to abnormal cell division, which might be result in cell apoptosis.

Wu et al. discovered that NUSAP1 also promotes cancer metastasis by activating the Hedgehog signaling pathway in astrocytoma (21). Gordon and his colleagues found that NUSAP1 enhances invasion and metastasis in prostate cancer (11). Whereas, whether NUSAP1 also promotes metastasis in HCC is unclear. We will clarify the above problems in our future study.

In conclusion, NUSAP1 is significantly upregulated in 
A
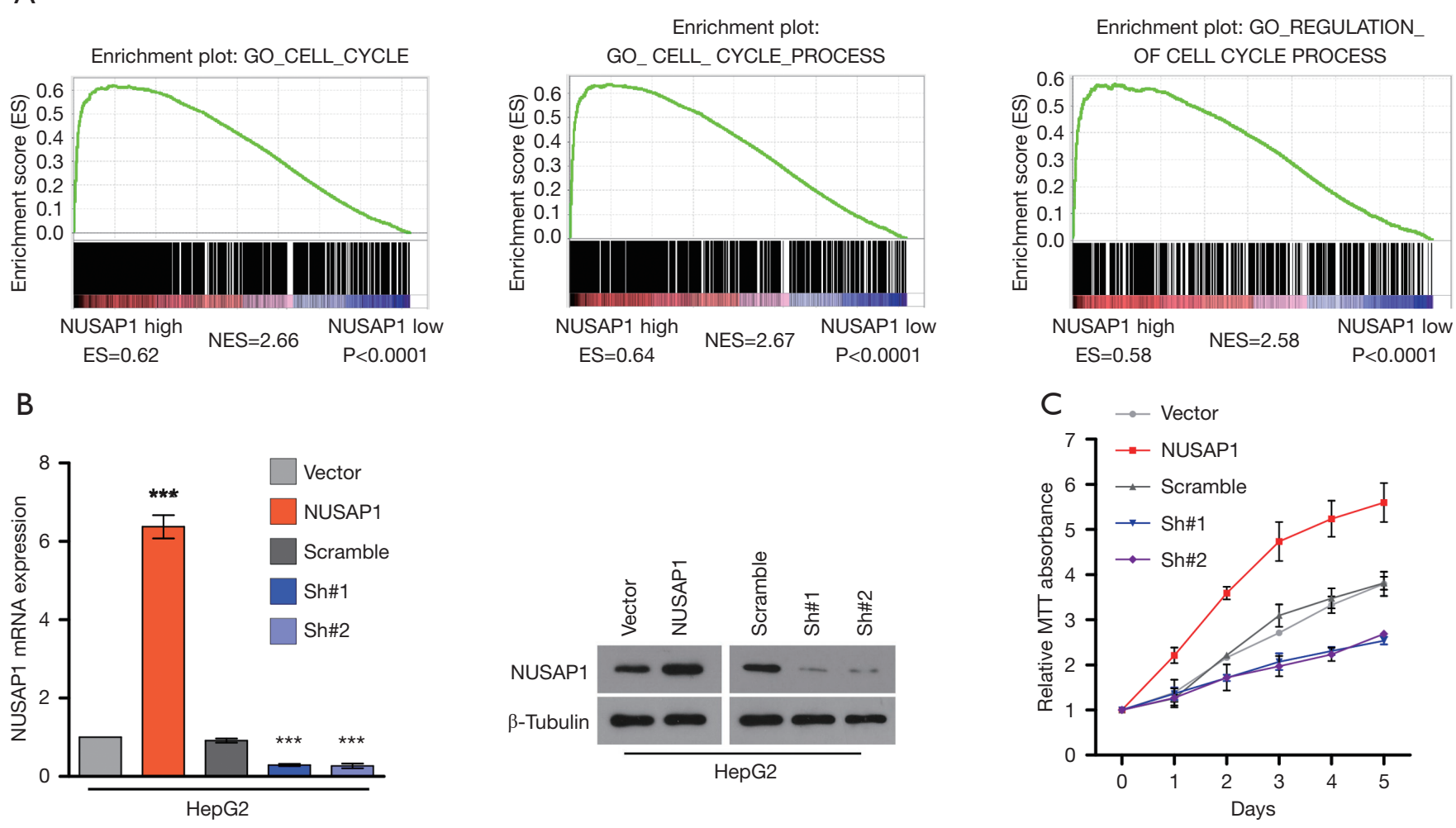

D
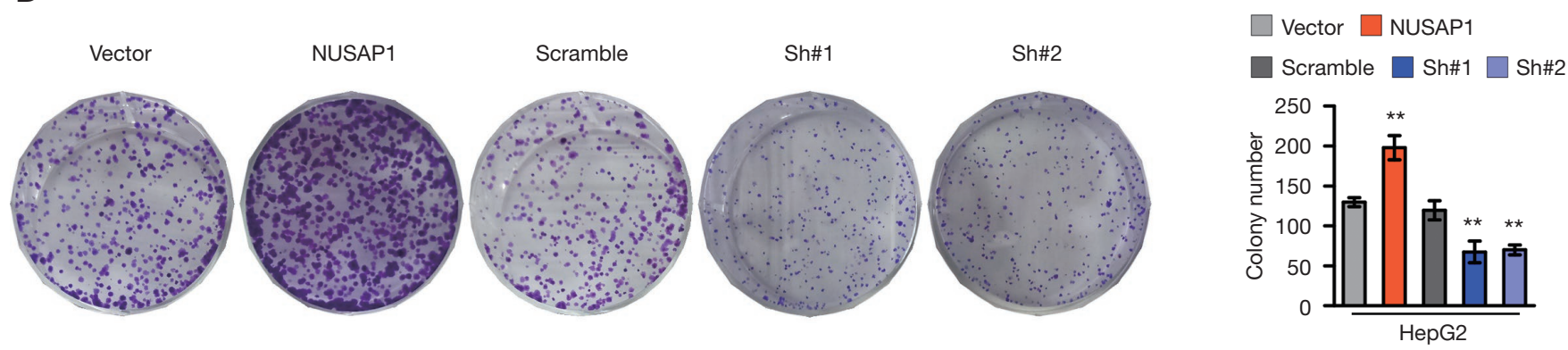

$E$

Vector

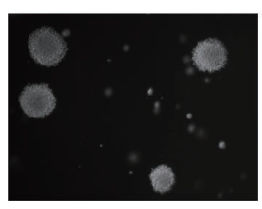

NUSAP1

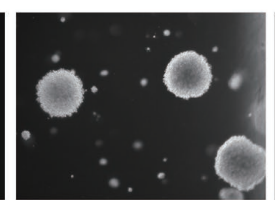

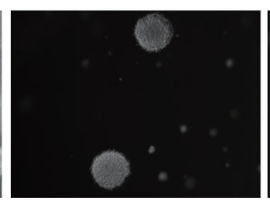
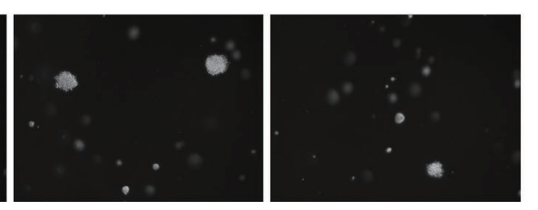

Figure 3 NUSAP1 can promote proliferation and in vitro tumorigenicity of HCC cells. (A) Gene set enrichment analysis (GSEA) illustrated that NUSAP1 might be involved in cell cycle progression using analyzing TCGA data. (B) qPCR (left panel) and western blotting assay (right panel) in indicated stable cell lines. (C) MTT assay showed that overexpression of NUSAP1 significantly promotes, while knockdown inhibits the proliferation of HCC cells. (D) Colony formation assay were used to detect the effect of NUSAP1 on HCC cell proliferation. (E) Anchorage-independent growth ability assay were performed to examine the effect of NUSAP1 on in vitro tumorigenicity of HCC cells. ${ }^{*} \mathrm{P}<0.05 ;{ }^{* *} \mathrm{P}<0.001 ;{ }^{* * *} \mathrm{P}<0.0001$. NUSAP1, nucleolar spindle associated protein $1 ;$ HCC, hepatocellular carcinoma. 
A

Enrichment plot: GO CELL CYCLE PHASE TRANSITION

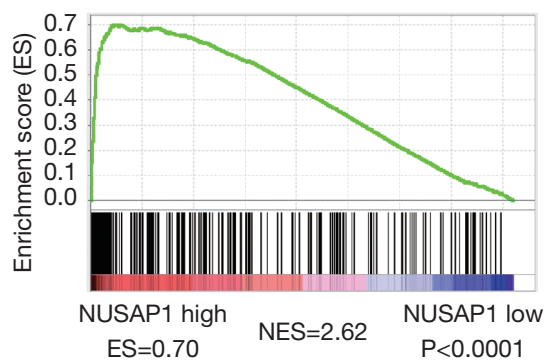

Enrichment plot: GO_CELL_ CYCLE_G1_S_PHASE_TRANSTIN

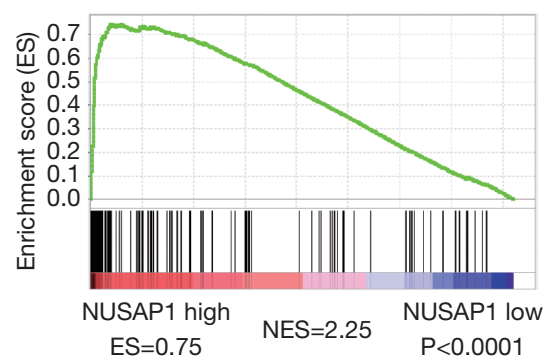

Enrichment plot: GO CELL CYCLE G2 M PHASE TRANSITION

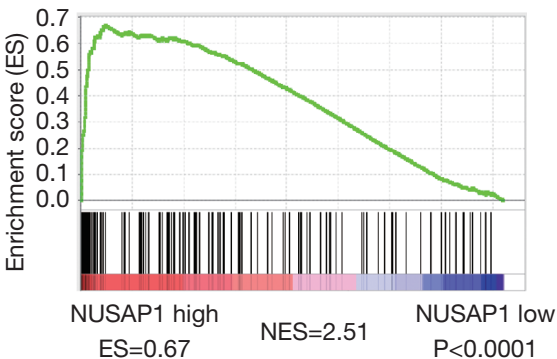

B
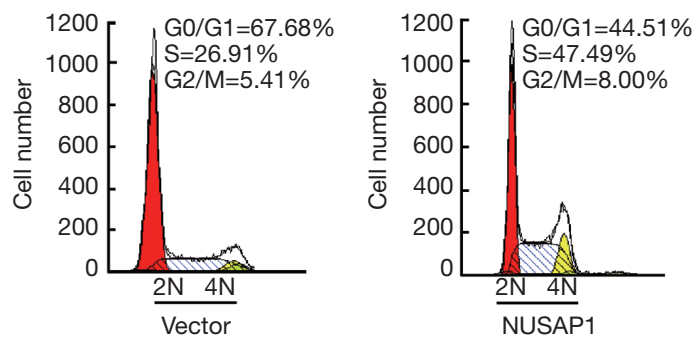

C
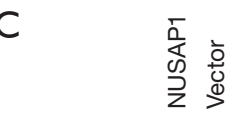

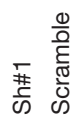
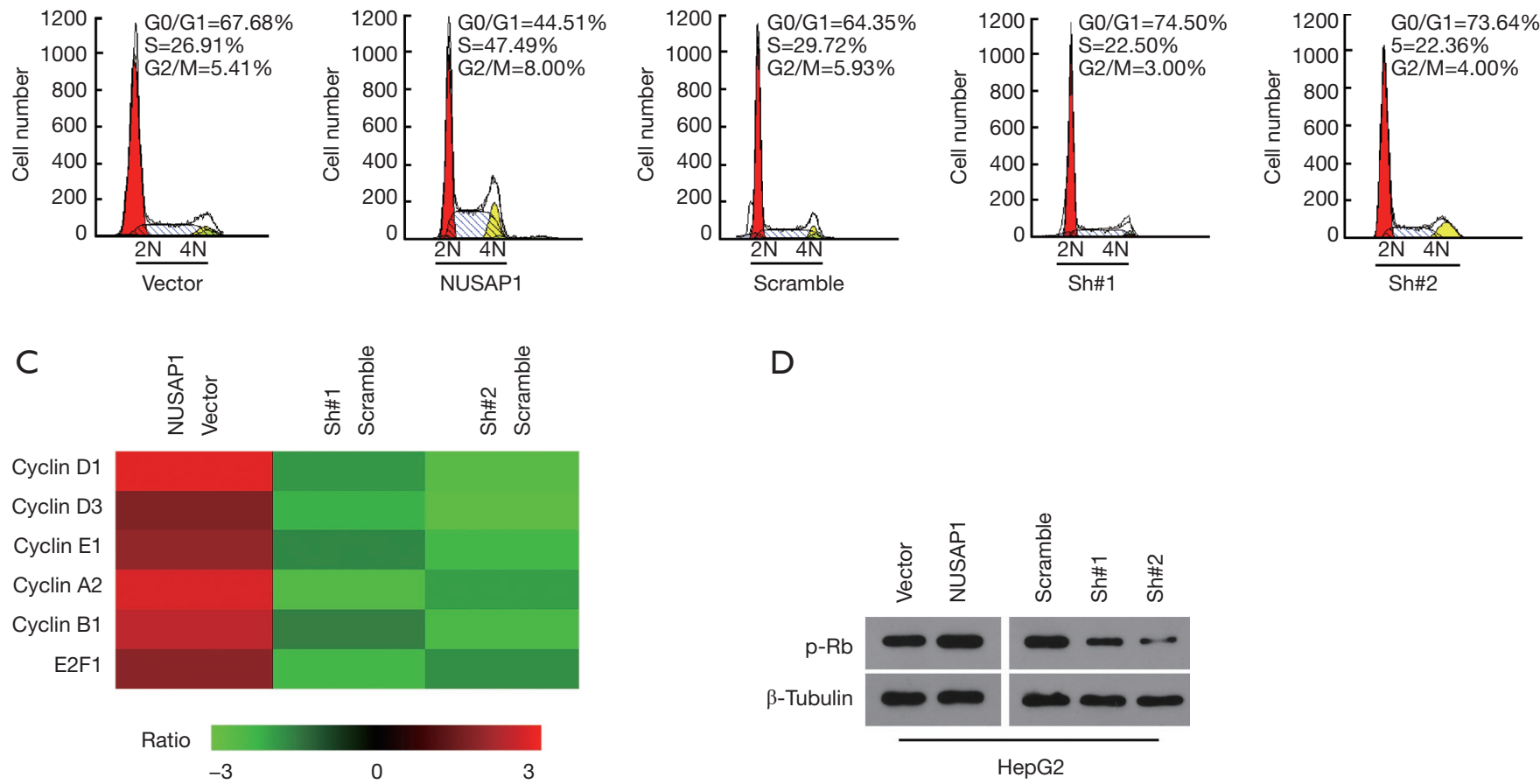

Figure 4 Overexpression of NUSAP1 facilitates cell cycle transition of HCC cells. (A) GSEA illustrated that NUSAP1 might be involved in G1/S phase and G2/M phase transition. (B) Flow cytometry assay demonstrated that overexpression of NUSAP1 increases the percentage of cells in S and G2/M phase and decreases in G0/G1 phase. (C) The heatmap for the expression of cyclin D1, cyclin D3, cyclin E1, cyclin $\mathrm{A} 2$, cyclin $\mathrm{B} 1$ and $\mathrm{E} 1 \mathrm{~F} 2$ in indicated cells. (D) The phosphorylation levels of $\mathrm{p}-\mathrm{Rb}$ in indicated cells. NUSAP1, nucleolar spindle associated protein 1; HCC, hepatocellular carcinoma; GSEA, gene set enrichment analysis.

HCC and associated with poor prognosis of patients with HCC. Further assay showed that NUSAP1 can promote cell cycle progression. Our study might provide a novel therapeutic prognostic factor for HCC.

\section{Acknowledgments}

Funding: This study was supported by the Development
Center for Medical Science and Technology National Health and Family Planning Commission of the People's Republic of China (No. W2017NJ07).

\section{Footnote}

Conflicts of Interest: All authors have completed the ICMJE uniform disclosure form (available at http://dx.doi. 
org/10.21037/tcr.2019.09.28). The authors have no conflicts of interest to declare.

Ethical Statement: The authors are accountable for all aspects of the work in ensuring that questions related to the accuracy or integrity of any part of the work are appropriately investigated and resolved. For research purpose, we acquired the informed consent of patient involved in the research. This study was approved by institutional ethics committee of Sun Yat-sen Memorial Hospital, Sun Yat-sen University (No. W2017NJ07). The research has been carried out in accordance with the Declaration of Helsinki (as revised in 2013) of the World Medical Association.

Open Access Statement: This is an Open Access article distributed in accordance with the Creative Commons Attribution-NonCommercial-NoDerivs 4.0 International License (CC BY-NC-ND 4.0), which permits the noncommercial replication and distribution of the article with the strict proviso that no changes or edits are made and the original work is properly cited (including links to both the formal publication through the relevant DOI and the license). See: https://creativecommons.org/licenses/by-nc-nd/4.0/.

\section{References}

1. Bray F, Ferlay J, Soerjomataram I, et al. Global cancer statistics 2018: GLOBOCAN estimates of incidence and mortality worldwide for 36 cancers in 185 countries. CA Cancer J Clin 2018;68:394-424.

2. Hanahan D, Weinberg RA. Hallmarks of cancer: the next generation. Cell 2011;144:646-74.

3. Morgan DO. Principles of CDK regulation. Nature 1995;374:131-4.

4. Nigg EA. Cyclin-dependent protein kinases: key regulators of the eukaryotic cell cycle. Bioessays 1995;17:471-80.

5. Harbour JW, Dean DC. Rb function in cell-cycle regulation and apoptosis. Nat Cell Biol 2000;2:E65-7.

6. Lim S, Kaldis P. Cdks, cyclins and CKIs: roles beyond cell cycle regulation. Development 2013;140:3079-93.

7. Hochegger H, Takeda S, Hunt T. Cyclin-dependent kinases and cell-cycle transitions: does one fit all? Nat Rev Mol Cell Biol 2008;9:910-6.

8. Chou HY, Wang TH, Lee SC, et al. Phosphorylation of NuSAP by Cdk1 regulates its interaction with microtubules in mitosis. Cell Cycle 2011;10:4083-9.

9. Mills CA, Suzuki A, Arceci A, et al. Nucleolar and spindleassociated protein 1 (NUSAP1) interacts with a SUMO
E3 ligase complex during chromosome segregation. J Biol Chem 2017;292:17178-89.

10. Li C, Xue C, Yang Q, et al. NuSAP governs chromosome oscillation by facilitating the Kid-generated polar ejection force. Nat Commun 2016;7:10597.

11. Gordon CA, Gong X, Ganesh D, et al. NUSAP1 promotes invasion and metastasis of prostate cancer. Oncotarget 2017;8:29935-50.

12. Okamoto A, Higo M, Shiiba M, et al. Down-Regulation of Nucleolar and Spindle-Associated Protein 1 (NUSAP1) Expression Suppresses Tumor and Cell Proliferation and Enhances Anti-Tumor Effect of Paclitaxel in Oral Squamous Cell Carcinoma. PLoS One 2015;10:e0142252.

13. Gulzar ZG, McKenney JK, Brooks JD. Increased expression of NuSAP in recurrent prostate cancer is mediated by E2F1. Oncogene 2013;32:70-7.

14. Chen L, Yang L, Qiao F, et al. High Levels of Nucleolar Spindle-Associated Protein and Reduced Levels of BRCA1 Expression Predict Poor Prognosis in Triple-Negative Breast Cancer. PLoS One 2015;10:e0140572.

15. Ye G, Sun G, Cheng Z, et al. p55PIK regulates alphafetoprotein expression through the NF-kappaB signaling pathway. Life Sci 2017;191:104-10.

16. Shi Y, Zhao Y, Zhang Y, et al. TNNT1 facilitates proliferation of breast cancer cells by promoting G1/S phase transition. Life Sci 2018;208:161-6.

17. Li B, Mao R, Liu C, et al. LncRNA FAL1 promotes cell proliferation and migration by acting as a CeRNA of miR-1236 in hepatocellular carcinoma cells. Life Sci 2018;197:122-9.

18. Lin J, Hou Y, Huang S, et al. Exportin-T Promotes Tumor Proliferation and Invasion in Hepatocellular Carcinoma. Mol Carcinog 2019;58:293-304.

19. Liu P, Chen B, Gu Y, et al. PNMA1, regulated by miR$33 \mathrm{a}-5 \mathrm{p}$, promotes proliferation and EMT in hepatocellular carcinoma by activating the $\mathrm{Wnt} /$ beta-catenin pathway. Biomed Pharmacother 2018;108:492-9.

20. Asghar U, Witkiewicz AK, Turner NC, et al. The history and future of targeting cyclin-dependent kinases in cancer therapy. Nat Rev Drug Discov 2015;14:130-46.

21. Wu X, Xu B, Yang C, et al. Nucleolar and spindle associated protein 1 promotes the aggressiveness of astrocytoma by activating the Hedgehog signaling pathway. J Exp Clin Cancer Res 2017;36:127.

Cite this article as: Hou S, Hua L, Wang W, Li M, Xu L. Nucleolar spindle associated protein 1 (NUSAP1) facilitates proliferation of hepatocellular carcinoma cells. Transl Cancer Res 2019;8(5):2113-2120. doi: 10.21037/tcr.2019.09.28 